\title{
Locking of symmetry breaking and topological phase in an interacting fermionic wire
}

\author{
Dan-Bo Zhang, ${ }^{1,2}$ Zhen Zheng, ${ }^{3}$ Y. X. Zhao $\odot,{ }^{4,5},{ }^{*}$ Qiang-Hua Wang,,${ }^{4,5, \dagger}$ and Z. D. Wang ${ }^{3,2, \$}$ \\ ${ }^{1}$ Guangdong Provincial Key Laboratory of Quantum Engineering and Quantum Materials, \\ GPETR Center for Quantum Precision Measurement, SPTE and Frontier Research Institute for Physics, \\ South China Normal University, Guangzhou 510006, China \\ ${ }^{2}$ The University of Hong Kong Shenzhen Institute of Research and Innovation, Shenzhen 518057, China \\ ${ }^{3}$ Department of Physics and HKU-UCAS Joint Institute for Theoretical and Computational Physics at Hong Kong, \\ The University of Hong Kong, Pokfulam Road, Hong Kong, China \\ ${ }^{4}$ National Laboratory of Solid State Microstructures \& School of Physics, Nanjing University, Nanjing 210093, China \\ ${ }^{5}$ Collaborative Innovation Center of Advanced Microstructures, Nanjing University, Nanjing 210093, China
}

(Received 6 November 2019; revised manuscript received 17 November 2019; accepted 24 December 2019; published 4 February 2020)

\begin{abstract}
We construct and study an intriguing model of one-dimensional interacting fermion wire, which is enforced to be in a topological phase by the spin-orbit locking structure in the interactions, regardless of the dimerization direction of the Peierls distortion, in sharp contrast to a conventional wisdom. Thorough analyses based upon the bosonization, the renormalization group technique, and the mean-field theory have been made. The novelty of being a pair-hopping fermionic wire lies in that symmetry breaking and the formation of (symmetry-protected) topological configurations are not independent, enriching our understanding of the interplay between topological phase and symmetry breaking. An experimentally feasible scheme is proposed for realizing the model with cold atoms and available techniques.
\end{abstract}

DOI: 10.1103/PhysRevResearch.2.013122

\section{INTRODUCTION}

During the past decade, profound understanding of symmetry-protected topological (SPT) phases have been one of main achievements in condensed matter physics [1-10]. For instance, for topological insulators and superconductors $[1,2,11,12]$, a complete classification with respect to time-reversal and charge-conjugate symmetry was established $[13,14]$. The new SPT paradigm has been enriching our understanding of many-body phases, which once was at large based on Landau's paradigm of symmetry breaking [15]. As the two paradigms follow essentially different ideologies developed naturally in physics, according to a conventional wisdom, they work in parallel for capturing features of many-body phases, that is symmetry-breaking processes determine the remaining symmetries of a system, and in turn SPT phases under the remaining symmetries enrich the phase diagram. In this paper, we present a 1D interacting fermion model, for which the translational symmetry breaking is locked with SPT phases protected by particle-hole symmetry, essentially due to the spin-orbit couplings in the interacting terms. The

\footnotetext{
*zhaoyx@nju.edu.cn

†qhwang@nju.edu.cn

‡wang@hku.hk
}

Published by the American Physical Society under the terms of the Creative Commons Attribution 4.0 International license. Further distribution of this work must maintain attribution to the author(s) and the published article's title, journal citation, and DOI. phenomenon indicates an insight that the relation between the two paradigms would be intricate, and more attention is needed to clarify their correlation.

It is a one-dimensional lattice model, which has the translational symmetry $\mathbf{Z}$ with the lattice constant $a$, the particle-hole symmetry $C$, and along with some other symmetries, such as the $U(1)$ symmetry and the global spin flipping symmetry. The interactions lead to the Peierls instability [16], and the lattice is distorted and dimerized, namely, the translational symmetry is reduced to be $2 \mathbf{Z}$ with the lattice constant $2 a$, leading to two degenerate ground states labeled by $\mathbf{Z}_{2} \cong$ $\mathbf{Z} / 2 \mathbf{Z}$, both having the particle-hole symmetry $C$. Intriguingly, the particular spin-orbit locking of the interactions enforces both directions of the dimerization to result in a $C$-symmetryprotected topological phase. Recall that for a conventional SuSchrieffer-Heeger (SSH) model $[17,18]$, only one direction of the dimerization leads to a topological phase while the other is trivial. The model is first solved by bosonization, where a more rigorous renomarlization group analysis is conducted. Guided by the identified order parameter, the solution is further examined by the mean-field method, and then confirmed by the DMRG analysis (not shown here). All the theoretical analyses suggest a simple explanation for why the system is always in the topological phase regardless of the dimerization direction. The present spinful model essentially consists of two SSH models, with each corresponding to a spin direction, and the particular spin-orbit interactions lock them to be out of phase, i.e., when one is in the topological phase the other is in the trivial phase. Then the change of the sign of the order parameter $M$ just switches the two models, and the overall system is always in the topological phase and has charge $1 / 2 \mathrm{~s}$ at the ends of the chain. 


\section{SSH MODEL REVISIT}

To prepare for addressing our more complicated model, we first introduce the well-understood SSH model briefly in accordance with our framework, which contains several major ingredients of our model as its prototype. After the dimerization of the $\mathrm{SSH}$ model, the two phases labeled by $\mathbf{Z}_{2}$ have the same reduced translational symmetry and $C$ symmetry but are topologically distinguishable, since only one of them has half charges located at two ends with natural open boundary conditions $[19,20]$. The corresponding topological invariant $\gamma \in$ $\mathbb{Z}_{2}$ is the $C$-symmetry quantized Berry phase of the valence bands, where in particular $\gamma \equiv 1 \bmod 2$ for the topological phase while $\gamma \equiv 0 \bmod 2$ for the trivial phase. Close to the free fermion critical point, the SSH model can be described by the boson theory, whose Lagrangian density reads

$$
\mathcal{L}_{\mathrm{SSH}}=\frac{1}{2 \pi}\left(\partial_{\mu} \varphi\right)^{2}+i \eta g \cos 2 \varphi,
$$

where $\eta=\eta_{L} \eta_{R}$ is the product of the Klein factors $\eta_{L / R}$, and $g$ is a coupling constant from the microscopic theory. Since the operator $\cos 2 \varphi$ has dimension 1 , it is highly relevant at long distances pinning $\varphi=0$ if ing $<0$ or $\varphi= \pm \pi / 2$ if ing $>0$. We identify ing $>0$ for the topological phase and ing $<0$ for the trivial one, since half charges are concentrated at two ends when $\varphi= \pm \pi / 2$ in the bulk, recalling that the ferminoic charge operator is $\rho=-\frac{1}{\pi} \partial_{x} \varphi$. It should not be surprising that $\eta=i$ or $-i$ may be related to the topology of the system, since the sign of $\eta$ is determined by the regularization scheme, while the topological invariant $\gamma$ also depends on the regularization in the continuous relativistic theory (detailed derivations can be found in Appendix A).

\section{Model and symmetries}

The proposed model Hamiltonian is given by

$$
\begin{aligned}
H= & \sum_{j \sigma}-t\left(c_{j \sigma}^{\dagger} c_{j+1 \sigma}+\text { H.c. }\right) \\
& +W_{1} \sum_{j}\left(c_{j \uparrow}^{\dagger} c_{j \downarrow}^{\dagger} c_{j+1 \downarrow} c_{j+1 \uparrow}+\text { H.c. }\right) \\
& +W_{2} \sum_{j}\left(c_{j \uparrow}^{\dagger} c_{j+1 \uparrow}^{\dagger} c_{j+1 \downarrow} c_{j \downarrow}+\text { H.c. }\right) .
\end{aligned}
$$

Here, in addition to a usual nearest-neighboring hopping term $t$, two other types of pair-hopping processes, $W_{1}$ term [21] and $W_{2}$ term [22], are considered. Before diving into the analysis of the model by bosonization, we address its several symmetries, which guide us in the thorough study of the model.

(1) First, the model has the lattice translational symmetry. (2) As advertised in the introduction, it has the particle-hole symmetry $C$, which is essential for the topological phase and acts as

$$
C c_{j} C^{-1}=(-1)^{j} c_{j}^{\dagger}, \quad C c_{j}^{\dagger} C^{-1}=(-1)^{j} c_{j} .
$$

The $C$ symmetry requires that $W_{1}$ and $W_{2}$ are real coupling constants. As highlighted in the Introduction, a novel phenomenon of the model is induced by the particular spin-orbit locking structure. Then we look into symmetries about the spin degree. (3) Although the full spin rotation symmetry is broken by the interaction terms, the model is invariant under spin-flipping $\sigma_{x}$, which flips two spin components, $c_{j \sigma} \rightarrow c_{j \bar{\sigma}}, c_{j \sigma}^{\dagger} \rightarrow c_{j \bar{\sigma}}^{\dagger}$. (4) Of course, this microscopic model satisfies the particle-number conservation, which is the consequence of $U(1)$ symmetry, $c_{\sigma} \rightarrow e^{-i \alpha} c_{\sigma}, c_{\sigma}^{\dagger} \rightarrow c_{\sigma}^{\dagger} e^{i \alpha}$. (5) The two spin components in the free Hamiltonian and the $W_{1}$ interaction term may follow independent global $U(1)$ transformation, namely, they are also invariant under the spin polarized $U^{S}(1)$ transformation $c_{\uparrow} \rightarrow e^{-i \beta} c_{\uparrow}, c_{\downarrow} \rightarrow e^{i \beta} c_{\downarrow}$ and H.c. However, the $U^{S}(1)$ symmetry is violated by the $W_{2}$ interaction, except for the special transformation, $c_{\uparrow} \rightarrow i c_{\uparrow}, c_{\downarrow} \rightarrow$ $-i c_{\downarrow}$ and H.c.. Combining the above special transformation with the spin flipping $\sigma_{x}$, the model has also the spin flipping symmetry $\sigma_{y}$ acting as $c_{\uparrow} \rightarrow i c_{\downarrow}, c_{\downarrow} \rightarrow-i c_{\uparrow}$ and H.c., which is just the spin flipping by $\sigma_{y}$. (6) As we shall see, the positivity of $W_{1}$ is essential in forming the topological phases after dimerization, but $W_{2}$ and $-W_{2}$ are related by the global spin rotation $R_{z}\left(\frac{\pi}{2}\right), c_{j} \rightarrow e^{i \frac{\sigma_{z}}{2} \frac{\pi}{2}} c_{j}, c_{j}^{\dagger} \rightarrow c_{j}^{\dagger} e^{-i \frac{\sigma_{z}}{2} \frac{\pi}{2}}$, while the $W_{1}$ term is preserved by $R_{z}\left(\frac{\pi}{2}\right)$. Thus, the model is symmetric under the combined transformation, $R_{z}\left(\frac{\pi}{2}\right)$ and $W_{2} \rightarrow-W_{2}$. Therefore, $W_{2}=0$ is a high symmetry point satisfying both $U^{S}(1)$ and $R_{z}\left(\frac{\pi}{2}\right)$ symmetry.

It may be illuminating to rewrite the interaction terms as

$$
H_{\mathrm{int}}=-g_{x} \sum_{j} O_{x}^{+}(j) O_{x}^{-}(j)-g_{y} \sum_{j} O_{y}^{+}(j) O_{y}^{-}(j) \text {, }
$$

where $O_{x}^{+}(j)=c_{j \uparrow}^{\dagger} c_{j+1 \downarrow}+$ H.c., $O_{x}^{-}(j)=c_{j \downarrow}^{\dagger} c_{j+1 \uparrow}+$ H.c. and $O_{y}^{+}(j)=i c_{j \uparrow}^{\dagger} c_{j+1 \downarrow}+$ H.c., $O_{y}^{-}(j)=-i c_{j \downarrow}^{\dagger} c_{j+1 \uparrow}+$ H.c., with $g_{x}=\frac{1}{2}\left(W_{1}+W_{2}\right), \quad g_{y}=\frac{1}{2}\left(W_{1}-W_{2}\right)$. We further define $O_{x}(j)=O_{x}^{+}(j)+O_{x}^{-}(j)=c_{j}^{\dagger} \sigma_{x} c_{j+1}+$ H.c., $\quad$ and $O_{y}(j)=O_{y}^{+}(j)+O_{y}^{-}(j)=c_{j}^{\dagger} \sigma_{y} c_{j+1}+$ H.c., which are related to one another by the spin rotation $R_{z}\left(\frac{\pi}{2}\right)$. Thus, if $O_{x}$ $\left(O_{y}\right)$ is the order parameter of the system for $W_{2}$, then $O_{y}\left(O_{x}\right)$ is that for $-W_{2}$.

\section{THE BOSON THEORY AND SYMMETRIES}

Let us assume that the system is close to the free fermion critical point, and thus we are able to make the continuum approximation as

$$
\frac{c_{j \sigma}^{\dagger}}{\sqrt{a}} \rightarrow \psi_{\sigma}^{\dagger}(x) \approx \psi_{L \sigma}^{\dagger}(x) e^{-i k_{F} x}+\psi_{R \sigma}^{\dagger}(x) e^{i k_{F} x} .
$$

The resulting continuous fermion theory is presented in Appendix $\mathrm{C}$ along with the derivation details.

Then, we use the bosonization to identify the relevant interactions in the model. We adopt the following dictionary of bosonization [23]:

$$
\psi_{L / R, \sigma}^{\dagger}(x)=\frac{\eta_{L / R, \sigma}}{\sqrt{2 \pi \alpha}} e^{i\left(\vartheta_{\sigma} \pm \varphi_{\sigma}\right)},
$$

where $\alpha$ is the short-distance cutoff and $\eta_{r, \sigma}$ are the Klein factors satisfying the Clifford algebra, $\left\{\eta_{r \sigma}, \eta_{r^{\prime} \sigma^{\prime}}\right\}=2 \delta_{r r^{\prime}} \delta_{\sigma \sigma^{\prime}}$. It is usually more convenient to work with the charge-spin separated basis, $\varphi_{c}=\frac{1}{\sqrt{2}}\left(\varphi_{\uparrow}+\varphi_{\downarrow}\right), \vartheta_{c}=\frac{1}{\sqrt{2}}\left(\vartheta_{\uparrow}+\vartheta_{\downarrow}\right), \varphi_{s}=$ $\frac{1}{\sqrt{2}}\left(\varphi_{\uparrow}-\varphi_{\downarrow}\right), \vartheta_{s}=\frac{1}{\sqrt{2}}\left(\vartheta_{\uparrow}-\vartheta_{\downarrow}\right)$. 
It is useful to elaborate the manifestation of symmetries in the boson theory. (1) The particle-hole symmetry, $C \psi^{\dagger}(j) C^{-1}=(-1)^{j} \psi(j)$, leads to $C \psi_{L / R}^{\dagger}(j) C^{-1}=\psi_{L / R}(j)$, $C \psi_{L / R}(j) C^{-1}=\psi_{L / R}^{\dagger}(j)$, and accordingly inverses all boson fields, $\varphi_{c / s} \rightarrow-\varphi_{c / s}, \vartheta_{c / s} \rightarrow-\vartheta_{c / s}$. (2) A unit lattice translation acts as $\psi_{L / R}^{\dagger}(x) \rightarrow \mp i \psi_{L / R}^{\dagger^{\prime}}(x)$ with $\psi^{\dagger^{\prime}}(x)=\psi^{\dagger}(x+a)$, which in boson version implies $\varphi_{\sigma} \rightarrow \varphi_{\sigma}-\pi / 2$, or equivalently $\varphi_{c} \rightarrow \varphi_{c}^{\prime}-\frac{\pi}{\sqrt{2}}$, while the other fields are only translated in coordinates. (3) The spin flipping $\sigma_{x}$ acts only on spin fields, $\varphi_{s} \rightarrow-\varphi_{s}, \vartheta_{s} \rightarrow-\vartheta_{s}$, and the Klein factors, $\eta_{r \sigma} \rightarrow$ $\eta_{r \bar{\sigma}}$. (4) For the $U(1)$ symmetry, $\vartheta_{c} \rightarrow \vartheta_{c}+\sqrt{2} \alpha$, and (5) for the $U^{S}(1)$ symmetry, $\vartheta_{s} \rightarrow \vartheta_{s}+\sqrt{2} \alpha_{S}$, in particular, for the special spinful transformation, $\vartheta_{s} \rightarrow \vartheta_{s}+\pi / \sqrt{2}$. (6) The spin rotation $R_{z}\left(\frac{\pi}{2}\right)$ corresponds to the transformation, $\vartheta_{s} \rightarrow$ $\vartheta_{s}+\pi / \sqrt{8}$.

The equivalent boson theory under the continuum approximation up to marginal terms has the nice feature of spincharge separation, namely, $H=H_{c}+H_{s}$, where

$$
\begin{array}{r}
H_{c}=H_{0, c}+\frac{2 g_{c}}{(2 \pi \alpha)^{2}} \int d x \cos \sqrt{8} \varphi_{c}, \\
H_{s}=H_{0, s}+\frac{2 g_{s}}{(2 \pi \alpha)^{2}} \int d x \cos \sqrt{8} \varphi_{s} \\
+\frac{2 h_{s}}{(2 \pi \alpha)^{2}} \int d x \cos \sqrt{8} \vartheta_{s},
\end{array}
$$

with $H_{0, v}=\frac{v_{v}}{2 \pi} \int d x\left[K_{v}\left(\partial_{x} \vartheta_{v}\right)^{2}+K_{v}^{-1}\left(\partial_{x} \varphi_{v}\right)^{2}\right]$, where the coupling constants are $v_{c}=v K_{c}$ with $v=2 t a, K_{c}=$ $\left(1-W_{1} / \pi t\right)^{1 / 2}, v_{s}=v K_{s}, K_{s}=\left(1+W_{1} / \pi t\right)^{1 / 2}$, and $g_{c}=$ $g_{s}=\eta\left(2 a W_{1}\right), h_{s}=\eta\left(4 a W_{2}\right)$, with $\eta=\eta_{L \uparrow} \eta_{L \downarrow} \eta_{R \uparrow} \eta_{R \downarrow}$.

It is observed that all aforementioned symmetries are preserved by the boson theory, and on the other hand, the form of the theory is strongly constrained by the symmetries as well. (1) All terms are even functions of boson fields, which conserves the particle-hole symmetry. (2) The lattice translational symmetry is preserved, since in the potential term, $\sqrt{8} \varphi_{c} \rightarrow \sqrt{8} \varphi_{c}+2 \pi$. (3) The spin flipping symmetry $\sigma_{x}$ is preserved, since all terms involving spin boson fields are even and $\eta$ is invariant under spin flipping. (4) The $U(1)$ symmetry is preserved since there is no potential terms of $\vartheta_{c}$. (5) If $W_{2}=0$, then the model has the spinful $U^{S}(1)$ symmetry, consistent with the fact that $h_{s}, h_{m}$, and $g_{m}$ vanish when $W_{2}=0$. If $W_{2} \neq 0$, then only the special symmetry $\vartheta_{s} \rightarrow \vartheta_{s}+\pi / \sqrt{2}$ is preserved. (6) Under the spin rotation $R_{z}(\pi / 2), \vartheta_{s}$ is converted to $\vartheta_{s}+\pi / \sqrt{8}$, which inverts the signs of all coupling constants proportional to $W_{2}, h_{s}, h_{m}$ and $g_{m}$, consistent with the fact that the model is symmetric under $W_{2} \rightarrow-W_{2}$.

\section{Order parameters and the renormalization group}

We now turn to investigate possible order parameters of the model. An order parameter for the bond spin density wave [24-26] (bSDW): $M_{x / y}=(-1)^{j}\left(c_{j}^{\dagger} \sigma_{x / y} c_{j+1}+\right.$ H.c. $)$. Under the continuum approximation, the corresponding boson operators are summarized in Table I. The pattern of sine and cosine functions in $M_{x}$ and $M_{y}$ in Table I reflects a fact that the spin rotation $R_{z}(\pi / 2)$ sending $\sigma_{x}$ to $\sigma_{y}$ leads to $\vartheta_{s} \rightarrow \vartheta_{s}+\frac{\pi}{2 \sqrt{2}}$, which exchanges $M_{x}$ and $M_{y}$. Recalling that $R_{z}(\pi / 2)$ also
TABLE I. Boson version of order parameters.

\begin{tabular}{ccc}
\hline \hline & $M_{x}$ & $M_{y}$ \\
\hline$\eta=+1$ & $\sin \sqrt{2} \varphi_{c} \sin \sqrt{2} \vartheta_{s}$ & $\sin \sqrt{2} \varphi_{c} \cos \sqrt{2} \vartheta_{s}$ \\
$\eta=-1$ & $\cos \sqrt{2} \varphi_{c} \cos \sqrt{2} \vartheta_{s}$ & $\cos \sqrt{2} \varphi_{c} \sin \sqrt{2} \vartheta_{s}$ \\
\hline \hline
\end{tabular}

sends $W_{2}$ to $-W_{2}$, we find if $M_{x}$ condensates for $W_{2}>0$, then $M_{y}$ condensates for $W_{2}<0$, and vise versa. Similar results can be obtained for the order parameter of the site-local spin density wave, $m_{x / y}=(-1)^{j} c_{j}^{\dagger} \sigma_{x / y} c_{j}$ (see Appendix E for details). These are the most possible order parameters, since density orders involving products of oscillations from conjugate fields, and pairing orders breaking the continuous $U(1)$ symmetry are impossible in $(1+1)$ dimensions.

At this stage, we proceed to identify the order parameters of the model by the renormalization group (RG) method. We here present the RG equations to the second order of coupling constants directly, while detailed derivations by operator product expansions can be found in Appendix D. Defining dimensionless coupling parameters as $y_{g c}=\frac{g_{c}}{\pi v_{c}}, y_{g s}=\frac{g_{s}}{\pi v_{s}}$ and $y_{h s}=\frac{h_{s}}{\pi v_{s}}$, the RG flow equations for charge fields are given as

$$
\begin{aligned}
& \frac{d y_{g c}}{d \ell}=\left(2-2 K_{c}\right) y_{g c}, \\
& \frac{d K_{c}}{d \ell}=-\frac{1}{2} K_{c}^{2} y_{g c}^{2},
\end{aligned}
$$

and those for spin fields are

$$
\begin{aligned}
& \frac{d y_{g s}}{d \ell}=\left(2-2 K_{s}\right) y_{g s}, \\
& \frac{d y_{h s}}{d \ell}=\left(2-2 K_{s}^{-1}\right) y_{h s}, \\
& \frac{d K_{s}}{d \ell}=\frac{1}{2}\left(y_{h s}^{2}-K_{s}^{2} y_{g s}^{2}\right),
\end{aligned}
$$

where the cutoff $\Lambda=\Lambda_{0} e^{-\ell}$ flows toward long distances by increasing $\ell$.

Solving the above flow equations numerically, we find that: (i) If $W_{1}<0,\left|W_{2}\right| \sim\left|W_{1}\right|$, then all potential energy terms are irrelevant, no matter $\eta= \pm 1$. (ii) If $W_{1}>0$ and $\left|W_{1}\right| \sim\left|W_{2}\right|$, then potential energy terms proportional to $g_{c}$ and $h_{s}$ are relevant, while the others are irrelevant, and $K_{c} \rightarrow 0, K_{s} \rightarrow \infty$, which means $\varphi_{c}$ and $\vartheta_{s}$ vary slowly at long distances, and are pinned at the minima of potential energies. There are totally four cases, for which $W_{2}>0$ and $W_{2}<0$ are related by the spin rotation from $\sigma_{x}$ to $\sigma_{y}$. (a) $W_{2}>0, \eta=1$, and the corresponding RG flows are illustrated in Fig. $1 . g_{c}>0, g_{s}>0$, leading to $\varphi_{c} \sim \pm \frac{\pi}{2 \sqrt{2}}$ and $\vartheta_{s} \sim \pm \frac{\pi}{2 \sqrt{2}}$. The nonvanishing order parameter is $M_{x}$. (b) $W_{2}>0, \eta=-1 . g_{c}<0, g_{s}<0$, leading to $\varphi_{c} \sim 0, \frac{\pi}{\sqrt{2}}$ and $\vartheta_{s} \sim 0, \frac{\pi}{\sqrt{2}}$. The nonvanishing order parameter is $M_{x}$. (c) $W_{2}<0, \eta=1 . g_{c}>0, g_{s}<0$, leading to $\varphi_{c} \sim \pm \frac{\pi}{2 \sqrt{2}}, \vartheta_{s} \sim 0, \frac{\pi}{\sqrt{2}}$. The nonvanishing order parameter is $M_{y}$. (d) $W_{2}<0, \eta=-1 . g_{c}<0, g_{s}>0$, leading to $\varphi_{c} \sim 0, \frac{\pi}{\sqrt{2}}$ and $\vartheta_{s} \sim \pm \frac{\pi}{2 \sqrt{2}}$. The nonvanishing order parameter is $M_{y}$. 


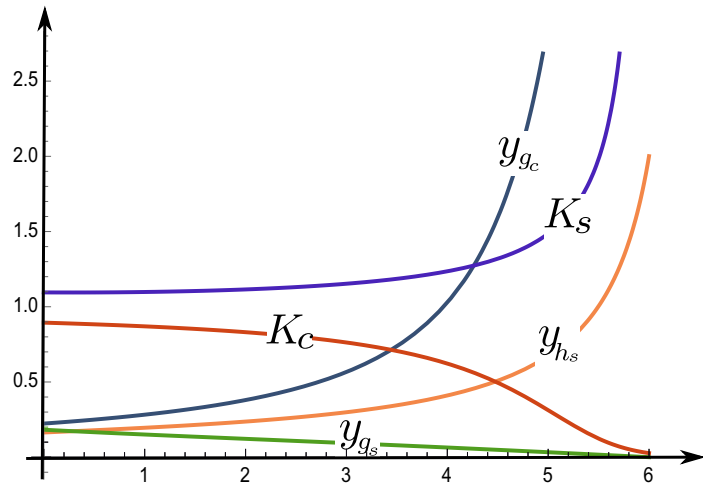

FIG. 1. RG flow of the coupling and Luttinger parameters. The bare parameters are chosen as $\frac{W_{1}}{\pi t}=0.2$ and $\frac{W_{2}}{\pi t}=0.9$ with $\eta=$ 1 , which corresponds to the initial values, $K_{c}=0.87, K_{s}=1.12$, $y_{g_{c}}=0.29, y_{g_{s}}=0.22$ and $y_{h_{s}}=0.22$.

We are mainly interested in the case (ii), which is summarized in Table II. In this case of $W_{1}>0$ and $\left|W_{2}\right| \sim\left|W_{1}\right|$, the original lattice translational symmetry $\mathbb{Z}$ of the lattice constant $a$ is always reduced to $2 \mathbb{Z}$ with the lattice constant $2 a$, and the two degenerate ground states correspond to $M_{x, y}= \pm\left|M_{x, y}\right|$, respectively, for the two directions of dimerization.

\section{MEAN-FIELD THEORY AND TOPOLOGICAL INVARIANT}

Since the order parameters have been identified by the RG analysis, let us make a mean-field analysis, which will fix $\eta$ in the above bosonization, analogous to the case of the SSH model that has been studied previously. We here present only details for $W_{2}>0$ with $M_{x} \neq 0$, since that of $W_{2}<0$ can be obtained by a global spin rotation $R_{z}(\pi / 2)$. We introduce $M_{x}^{ \pm}(j)=(-1)^{j}\left\langle O_{x}^{ \pm}(j)\right\rangle$, and $M_{x}=M_{x}^{+}+M_{x}^{-}$. Noting that the condensation of $M_{x}$ preserves the spin flipping symmetry $\sigma_{x}$, but breaks $\sigma_{y}$, we have $M_{x}^{+}=M_{x}^{-}$, since $M_{x}^{ \pm}$are related by the spin flipping $\sigma_{x}$. Introducing $M(j)=-g_{x} M_{x}^{+}=$ $-g_{x}(-1)^{j}\left\langle O_{x}^{+}(j)\right\rangle$, the mean-field Hamiltonian is written as

$$
H_{M F}=\sum_{j} c_{j}^{\dagger}\left[-t \sigma_{0}+(-1)^{j} M \sigma_{x}\right] c_{j+1}+\text { H.c. }
$$

where we have ignored the second term in Eq. (4) which is irrelevant. The self-consistence of the mean-field theory is checked in Appendix F. The second term from bSDW indicates the breaking of translational symmetry from $\mathbf{Z}$ to $2 \mathbf{Z}$, and it is the spin-dependency herein that ensures the topological invariant always nontrivial regardless of the sign of the bSDW order parameter $M$ (see Appendix F for detailed

TABLE II. Pinned bosonic fields and order parameters.

\begin{tabular}{cccccc}
\hline \hline & $\eta=1$ & $\eta=-1$ & Order & PHS \\
\hline$W_{2}>0$ & $\varphi_{c} \sim \pm \frac{\pi}{2 \sqrt{2}}$ & $\varphi_{c}$ & $\sim 0, \frac{\pi}{\sqrt{2}}$ & $M_{x}$ & $\hat{C}=\tau_{3} \hat{\mathcal{K}} \hat{I}$ \\
& $\vartheta_{s} \sim \pm \frac{\pi}{2 \sqrt{2}}$ & $\vartheta_{s} \sim 0, \frac{\pi}{\sqrt{2}}$ & & \\
$W_{2}<0$ & $\varphi_{c} \sim \pm \frac{\pi}{2 \sqrt{2}}$ & $\varphi_{c} \sim 0, \frac{\pi}{\sqrt{2}}$ & $M_{y}$ & $\hat{C}=\sigma_{3} \tau_{3} \hat{\mathcal{K} \hat{I}}$ \\
& $\vartheta_{s} \sim 0, \frac{\pi}{\sqrt{2}}$ & $\vartheta_{s} \sim \pm \frac{\pi}{2 \sqrt{2}}$ & & \\
\hline \hline
\end{tabular}

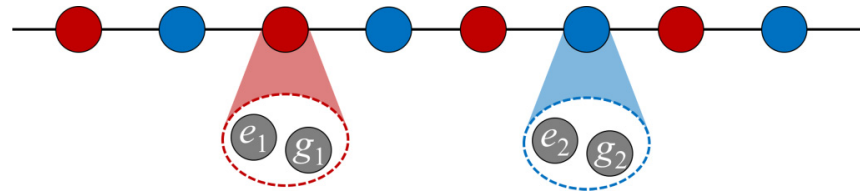

FIG. 2. The cold-atom lattice consisting of two sublattices. Atoms in $\left|g_{1}\right\rangle$ and $\left|e_{1}\right\rangle\left(\left|g_{2}\right\rangle\right.$ and $\left.\left|e_{2}\right\rangle\right)$ reside in the red (blue) sites.

derivations). In addition, due to the symmetry structure, our analysis for topological invariant is complete in the whole parameter space, which can be seen from Appendix B.

\section{COLD-ATOM REALIZATION}

We now turn to realizing the model of Eq. (2) by ultracold fermionic atoms trapped in a 1D optical lattice, where notably the hopping and interaction strengths are all independently tunable. Since every interaction term is composed of four operators distinguishable by lattice sites and spins, we shall accordingly choose four atomic states $\left|\lambda_{s}\right\rangle$, where $\lambda=e, g$ labeling two atoms, ${ }^{1} S_{0}$ and ${ }^{3} P_{1}$, and $s=1,2$ labeling the spin states $\mid 5 / 2,-5 / 2$ or $-3 / 2\rangle$ of the two atoms. Then it is desirable to construct a spin-dependent optical lattice consisting of two sublattices as illustrated in Fig. 2, where atoms of $\left|\lambda_{1}\right\rangle\left(\left|\lambda_{2}\right\rangle\right)$ are trapped in a sublattice by the potential $V_{1}(x)=$ $V_{L} \sin ^{2}\left(k_{L} x\right) \quad\left[V_{2}(x)=V_{L} \cos ^{2}\left(k_{L} x\right)\right]$ with $k_{L}=\pi / 2 a$. The spin dependence is obtained by adding an electro-optical monitor to the counter-propagating lasers that generates the lattice potential [27]. With cold atoms trapped in the optical lattice of Fig. 2, we now assign the correspondences: $g_{1} \rightarrow c_{j \downarrow}$, $e_{1} \rightarrow c_{j \uparrow}, g_{2} \rightarrow c_{j+1 \uparrow}, e_{2} \rightarrow c_{j+1 \downarrow}$, and proceed to realize the Hamiltonian of Eq. (2). First, the free part of Eq. (2), which corresponds to the tunnelings between adjacent optical lattice sites, can be easily realized via the laser-assisted tunneling technique [28,29]. For the two interaction terms, the $W_{2}$ term can be conventionally generated via the magnetic Feshbach resonance in alkaline-earth(-like) atoms [30,31]. And to individually tune the two interacting strengths, we engineer the $W_{1}$ term by the optical Feshbach resonance [32]. Particularly, we assign $|e, g\rangle$ and $\left|e_{1}, e_{2}\right\rangle$ as the open and closed channels, respectively, and then two adjacent open channels in the optical lattice sites can be coupled by two circularly polarized Raman lasers with $\left|e_{1}, e_{2}\right\rangle$ as the intermediate states. Note that the transition between the two open channels $\left|g_{1}, e_{2}\right\rangle$ and $\left|g_{2}, e_{1}\right\rangle$ is prohibited because of the selection rule in the presence of circularly polarized lasers. The technical details are included in Appendix G.

\section{BRIEF SUMMARY}

We have constructed an intriguing one-dimensional interacting fermionic model that exhibits an unconventional class of topological phenomena, namely, the system is enforced into an SPT phase under symmetry breaking, suggesting that the relationship between symmetry breaking and the SPTphase theory be intricate. In-depth analyses based upon the bosonization, the RG technique, and the mean-field theory have been conducted thoroughly, elucidating the exotic interplay between topological characters and symmetries un- 
ambiguously. Finally, it is remarkable that we have worked out how to emulate the model by fermionic cold-atoms with available experimental techniques.

\section{ACKNOWLEDGMENTS}

This work was supported by the GRF of Hong Kong (Grants No. HKU173309/16P and No. HKU173057/17P), CRF (Grant No. C6005-17G) of Hong Kong, the NKRDP of China (Grant No. 2016YFA0301802), the Key R\&D Program of Guangdong province (Grant No. 2019B030330001), and the NSFC (Grant No. 11874201 and 11574134).

\section{APPENDIX A: BOSONIZATION OF THE SSH MODEL}

The topological feature of SSH model is captured by the one-dimensional massive Dirac model,

$$
\mathcal{L}_{D}=\bar{\Psi} i \gamma^{\mu} \partial_{\mu} \Psi+m \bar{\Psi} \Psi
$$

where

$$
\gamma^{0}=\left(\begin{array}{cc}
0 & -i \\
i & 0
\end{array}\right), \quad \gamma^{1}=\left(\begin{array}{ll}
0 & i \\
i & 0
\end{array}\right) .
$$

The model has the particle-hole symmetry

$$
\psi \rightarrow \psi^{\dagger}, \quad \psi^{\dagger} \rightarrow \psi .
$$

This can be explicitly seen from the Hamiltonian in momentum space,

$$
h(k)=\gamma^{0} \gamma^{1} k-\left(m-\epsilon k^{2}\right) \gamma^{0},
$$

for which the particle-hole symmetry is represented by $\hat{C}=$ $\hat{\mathcal{K}} \hat{I}$ with $\hat{I}$ being the inversion of momentum and $\hat{\mathcal{K}}$ the complex conjugate. Here, an infinitesimal term $\epsilon k^{2} \gamma^{0}$ has been introduced as the regularization for large momenta. It is clear that the massive Dirac model is in the class $D$ with $\mathbb{Z}_{2}$ classification, and the corresponding topological number is given by

$$
N \equiv \frac{1}{2}[\operatorname{sgn}(m)+\mathbf{s g n}(\epsilon)] \bmod 2 .
$$

For $N \equiv 1 \bmod 2$, there are half charges at two ends for an open system. If the Dirac model is the low-energy effective theory of a lattice model, then the complete lattice model provides the effective theory a natural lattice regularization corresponding to a specific $\epsilon$.

The bosonic version of the kinetic term is just the free boson, and the mass term is

$$
\begin{aligned}
\mathcal{V} & =-m \bar{\Psi} \Psi=i m\left(\psi^{\dagger} \bar{\psi}-\bar{\psi}^{\dagger} \psi\right) \\
& =\frac{i \eta_{L} \eta_{R}}{\pi \alpha} m \cos 2 \varphi .
\end{aligned}
$$

$\eta_{L} \eta_{R}$ is antihermitian and setting its value to $\pm i$ is just a convention. Without loss of generality we adopt $\eta_{L} \eta_{R}=-i$, which gives the bosonization of the Dirac model,

$$
\mathcal{L}=\frac{1}{2 \pi K} \partial_{\mu} \varphi \partial^{\mu} \varphi-g \cos (2 \varphi),
$$

with $K=1$ and $g=\frac{m}{\pi \alpha}$. As being derived by operator product expansion in Sec. D, the renormalization group equations are

$$
\frac{d K}{d \ell}=-c g^{2} K^{2}, \quad \frac{d g}{d \ell}=(2-K) g,
$$

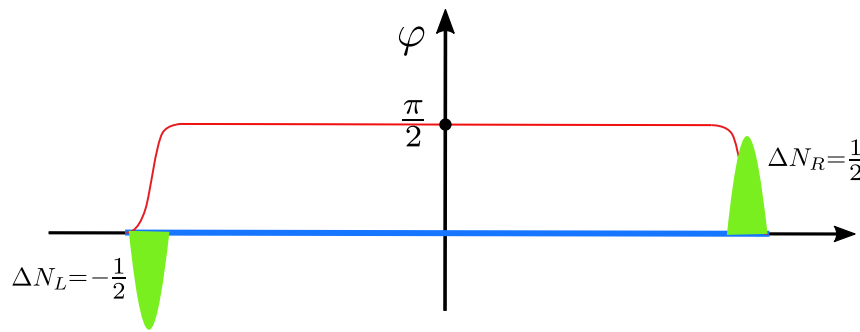

FIG. 3. half charges at the ends of a gapped fermion wire with $\varphi=\frac{\pi}{2}$.

which means the term $\mathcal{H}_{m}=g \cos (2 \varphi)$ dominates toward infrared. If the bare parameter $m<0$, then the $\varphi$ is pinned to $\varphi=0$ close to the ground state, while starting with $m>0$, $\varphi$ is pinned to $\varphi=-\frac{\pi}{2}$ or $\frac{\pi}{2}$. When considering a closed wire, we cannot physically distinguish $\varphi=-\frac{\pi}{2}$ from $\varphi=\frac{\pi}{2}$. But for an open wire if we connect its ends to wires with $m<0$ and $\varphi=0$, there are net half charges concentrated at the ends. When $\varphi=\frac{\pi}{2}$, as shown in Fig. 3, the net fermionic number at the two ends can be computed by as

$$
\Delta N_{L / R}=-\frac{1}{\pi} \int_{L / R} \partial_{x} \varphi=\mp \frac{1}{2} .
$$

When $\varphi=-\frac{\pi}{2}$, the net fermionic numbers are inversed.

Thus, we conclude that $m>0$ and $m<0$ correspond to different phases, where one is topological and the other is trivial. The topological feature is the existence of half fermionic numbers at the ends. Deciding which one is topological depends on the ultraviolet regularization in the fermionic theory. Here in the bosonization procedure, this depends on our convention for Klein factors, which is completely determined if we have a microscopic lattice model. Particularly, $\eta_{L} \eta_{R}=i$ or $-i$ corresponds to $\epsilon>0$ or $\epsilon<0$ in Eq. (A4).

\section{APPENDIX B: SYMMETRY TRANSFORMATIONS}

Symmetry transformations of fields discussed in the main text are tabulated in Table III.

\section{APPENDIX C: CONTINUOUS APPROXIMATION}

Noting that

$$
\sum_{j} a(\cdots) \rightarrow \int d x(\cdots),
$$

the free part can be approximated continuously as

$$
\begin{aligned}
H_{0} & =\sum_{j \sigma}-t\left(c_{j \sigma}^{\dagger} c_{j+1 \sigma}+\text { H.c. }\right) \\
& \rightarrow-i v \sum_{\sigma} \int d x\left(\psi_{L \sigma}^{\dagger} \partial_{x} \psi_{L \sigma}-\psi_{R \sigma}^{\dagger} \partial_{x} \psi_{R \sigma}\right),
\end{aligned}
$$

with $v=2 t a$.

The first interacting term is approximated continuously as

$$
H_{W_{1}} \rightarrow a W_{1} \int d x\left(\psi_{\uparrow}^{\dagger} \psi_{\downarrow}^{\dagger} \psi_{\downarrow}^{\prime} \psi_{\uparrow}^{\prime}+\text { H.c. }\right),
$$


TABLE III. Table of symmetry transformations. Each empty entry indicates the field is invariant under the corresponding symmetry transformation except for translational symmetry. For translational symmetry, the spatial coordinates of every field are translated for a lattice spacing, and only additional operations are displayed.

\begin{tabular}{lcccccc}
\hline \hline & $T$ & PHS & $\sigma_{x}$ & $U(1)$ & $U^{S}(1)$ & $R_{z}\left(\frac{\pi}{2}\right)$ \\
\hline$c_{j \sigma}^{\dagger}$ & $c_{j+1}^{\dagger}$ & $(-1)^{j} c_{j}^{\dagger}$ & $c_{j \bar{\sigma}}^{\dagger}$ & $c_{j}^{\dagger} e^{i \alpha}$ & $c_{j}^{\dagger} e^{i \alpha \sigma_{z}}$ & $c_{j}^{\dagger} e^{-i \frac{\sigma_{z}}{2} \frac{\pi}{2}}$ \\
$\psi_{L / R, \sigma}^{\dagger}$ & $\mp i \psi_{L / R}^{\dagger}$ & $\psi$ & $\psi_{\bar{\sigma}}^{\dagger}$ & $\psi^{\dagger} e^{i \alpha}$ & $\psi^{\dagger} e^{i \alpha \sigma_{z}}$ & $\psi^{\dagger} e^{-i \frac{\sigma_{z}}{2} \frac{\pi}{2}}$ \\
$\varphi_{c / s}$ & $\varphi_{c}-\frac{\pi}{\sqrt{2}}$ & $-\varphi$ & $-\varphi_{s}$ & & & \\
$\vartheta_{c / s}$ & & $-\vartheta$ & $-\vartheta_{s}$ & $\vartheta_{c}+\sqrt{2} \alpha$ & $\vartheta_{s}+\sqrt{2} \alpha$ & $\vartheta_{s}+\pi / \sqrt{8}$ \\
\hline \hline
\end{tabular}

with $\psi^{\prime}(x)=\psi(x+a)$. Noting that $\psi$ has dimension $1 / 2$, we expand the Hamiltonian to dimension 4, namely,

$$
H_{W_{1}}=a W_{1} \int d x O_{W_{1}}^{2}+O_{W_{1}}^{4}+\mathcal{O}\left(\Lambda^{6}\right)
$$

Since

$$
\begin{aligned}
\psi_{\uparrow}^{\dagger} \psi_{\uparrow}^{\prime}= & i\left(\rho_{L \uparrow}-\rho_{R \uparrow}\right) \\
& +i\left(\psi_{R \uparrow}^{\dagger} \psi_{L \uparrow}^{\prime} e^{2 i k_{F} x}-\psi_{L \uparrow}^{\dagger} \psi_{R \uparrow}^{\prime} e^{-2 i k_{F} x}\right)+\mathcal{O}(a),
\end{aligned}
$$

we proceed

$$
\begin{aligned}
O_{W_{1}}^{2}= & \psi_{\uparrow}^{\dagger} \psi_{\downarrow}^{\dagger} \psi_{\downarrow} \psi_{\uparrow}+\text { H.c. } \\
= & -\left(\rho_{L \uparrow}-\rho_{R \uparrow}\right)\left(\rho_{L \downarrow}-\rho_{R \downarrow}\right) \\
& +\psi_{R \uparrow}^{\dagger} \psi_{L \uparrow} \psi_{L \downarrow}^{\dagger} \psi_{R \downarrow}+\psi_{L \uparrow}^{\dagger} \psi_{R \uparrow} \psi_{R \downarrow}^{\dagger} \psi_{L \downarrow} \\
& -\psi_{R \uparrow}^{\dagger} \psi_{L \uparrow} \psi_{R \downarrow}^{\dagger} \psi_{L \downarrow}-\psi_{L \uparrow}^{\dagger} \psi_{R \uparrow} \psi_{L \downarrow}^{\dagger} \psi_{R \downarrow}+\text { H.c. } \\
= & -2\left(\rho_{L \uparrow}-\rho_{R \uparrow}\right)\left(\rho_{L \downarrow}-\rho_{R \downarrow}\right) \\
& +2\left(\psi_{R \uparrow}^{\dagger} \psi_{L \uparrow} \psi_{L \downarrow}^{\dagger} \psi_{R \downarrow}+\text { H.c. }\right) \\
& -2\left(\psi_{R \uparrow}^{\dagger} \psi_{L \uparrow} \psi_{R \downarrow}^{\dagger} \psi_{L \downarrow}+\text { H.c. }\right) .
\end{aligned}
$$

The second interaction term $H_{W_{2}}$ corresponds to

$$
\begin{aligned}
H_{W_{2}} & \rightarrow a W_{2} \int d x\left(\psi_{\uparrow}^{\dagger} \psi_{\uparrow}^{\prime \dagger} \psi_{\downarrow}^{\prime} \psi_{\downarrow}+\text { H.c. }\right) \\
& =a W_{2} \int d x O_{W_{2}}^{2}+O_{W_{2}}^{4}+\mathcal{O}\left(\Lambda^{6}\right) .
\end{aligned}
$$

With

$$
\begin{aligned}
\psi_{\uparrow}^{\dagger} \psi_{\downarrow}= & \psi_{L \uparrow}^{\dagger} \psi_{L \downarrow}+\psi_{R \uparrow}^{\dagger} \psi_{R \downarrow} \\
& +\psi_{R \uparrow}^{\dagger} \psi_{L \downarrow} e^{2 i k_{F} x}+\psi_{L \uparrow}^{\dagger} \psi_{R \downarrow} e^{-2 i k_{F} x}
\end{aligned}
$$

and

$$
\begin{aligned}
\psi_{\uparrow}^{\prime \dagger} \psi_{\downarrow}^{\prime}= & \psi_{L \uparrow}^{\prime \dagger} \psi_{L \downarrow}^{\prime}+\psi_{R \uparrow}^{\prime \dagger} \psi_{R \downarrow}^{\prime} \\
& -\psi_{R \uparrow}^{\prime \dagger} \psi_{L \downarrow}^{\prime} e^{2 i k_{F} x}-\psi_{L \uparrow}^{\prime \dagger} \psi_{R \downarrow}^{\prime} e^{-2 i k_{F} x},
\end{aligned}
$$

we have

$$
O_{W_{2}}^{2}=4 \psi_{L \uparrow}^{\dagger} \psi_{L \downarrow} \psi_{R \uparrow}^{\dagger} \psi_{R \downarrow}+\text { H.c. }
$$

\section{APPENDIX D: RENORMALIZATION BY OPERATOR PRODUCT EXPANSION}

We assume that $S_{0}[\phi]$ describes a critical point and shall discuss perturbations that may drive the system away from the critical point. At the critical point, it is natural to assume that each perturbation term has a definite scaling dimension, namely,

$$
S[\phi]=S_{0}[\phi]+\sum_{j} g_{j} \int d^{d} x O_{j}(x),
$$

where each $O_{j}(x)$ is a local operator with scaling dimension $\Delta_{j}$. Without loss of generality, we assume that the set of $O_{j}(x)$ is closed under operator product expansion, which is given by

$$
O_{j}\left(x^{\prime}\right) O_{k}(x)=\sum_{l} \frac{1}{\left|x^{\prime}-x\right|^{\Delta_{j}+\Delta_{k}-\Delta_{l}}} C_{j k}^{l} O_{l}(x) .
$$

Then,

$$
g_{l}^{\prime}=g_{l} L^{2-\Delta_{l}}-\frac{1}{2} \Omega_{d} \sum_{j k} C_{j k}^{l} g_{j} g_{k}\left\{\begin{array}{c}
\frac{L^{2-\Delta_{j}-\Delta_{k}+\Delta_{l}}}{d-\Delta_{j}-\Delta_{k}+\Delta_{l}} \\
\ln L
\end{array}\right\} L^{2-\Delta_{l}},
$$

with $L=e^{\ell}$, which gives the renormalization of the theory to the second order of coupling constants.

For the bosonization theories, we may need to following OPEs.

$$
\begin{aligned}
e^{i a \phi(w)} e^{-i b \phi(z)} \sim & \frac{1}{(w-z)^{a b}} e^{i(a-b) \phi(z)} \\
& +\frac{i a}{(w-z)^{a b-1}} \partial \phi(z) e^{i(a-b) \phi(z)}, \\
\partial \phi(w) e^{i a \phi(z)} \sim & -\frac{i a}{w-z} e^{i a \phi(z)}+\partial \phi(z) e^{i a \phi(z)} .
\end{aligned}
$$

\section{Massive free Dirac fermion}

The boson version of the massive free Dirac fermion is

$$
\mathcal{L}=\frac{1}{2 \pi} \partial_{\mu} \varphi \partial^{\mu} \varphi+g_{0} \partial_{\mu} \varphi \partial^{\mu} \varphi+g_{1} \cos (2 \varphi) .
$$

Define

$$
O_{0}(x)=\partial_{\mu} \varphi \partial^{\mu} \varphi(x)=\partial \phi(z) \bar{\partial} \bar{\phi}(\bar{z}),
$$

$$
O_{1}(x)=\cos (2 \varphi(x))=\frac{1}{2}\left\{e^{i[\phi(z)+\bar{\phi}(\bar{z})]}+\text { H.c. }\right\},
$$

for which $\Delta_{0}=2$ and $\Delta_{1}=1$. Then the relevant OPEs are

$$
\begin{aligned}
& O_{1}(w) O_{1}(z) \sim \frac{1}{2} \frac{1}{|w-z|^{2}}-\frac{1}{2} O_{0}(z), \\
& O_{0}(w) O_{1}(z) \sim \frac{-1}{|w-z|^{2}} O_{1}(z) .
\end{aligned}
$$


Thus, the RG equations are

$$
\frac{d g_{0}}{d \ell}=\frac{\pi}{2} g_{1}^{2}, \quad \frac{d g_{1}}{d \ell}=g_{1}+2 \pi g_{0} g_{1} .
$$

We introduce

$$
\frac{1}{2 \pi K}=\frac{1}{2 \pi}+g_{0},
$$

then the $\mathrm{RG}$ equations are rewritten as

$$
\frac{d K}{d \ell}=-\pi^{2} K^{2} g_{1}^{2}, \quad \frac{d g_{1}}{d \ell}=(2-K) g_{1} .
$$

\section{Spinful field}

Consider the Lagrangian,

$$
\begin{aligned}
\mathcal{L}= & \frac{1}{2 \pi} \partial_{\mu} \varphi \partial^{\mu} \varphi+g_{0} \partial_{\mu} \varphi \partial^{\mu} \varphi \\
& +g_{1} \cos (2 \sqrt{2} \varphi)+g_{2} \cos (2 \sqrt{2} \vartheta),
\end{aligned}
$$

for which all perturbation terms are marginal with $\Delta_{i}=2$, $i=0,1,2$. Introducing

$$
\begin{aligned}
& O_{0}(x)=\partial_{\mu} \varphi \partial^{\mu} \varphi(x)=\partial \phi(z) \bar{\partial} \bar{\phi}(\bar{z}) \\
& O_{1}(x)=\cos (2 \sqrt{2} \varphi)=\frac{1}{2}\left(e^{i(\sqrt{2} \phi(z)+\sqrt{2} \bar{\phi}(\bar{z}))}+\text { H.c. }\right), \\
& O_{2}(x)=\cos (2 \sqrt{2} \vartheta)=\frac{1}{2}\left(e^{i(\sqrt{2} \phi(z)-\sqrt{2} \bar{\phi}(\bar{z}))}+\text { H.c. }\right),
\end{aligned}
$$

we can derive the following relevant OPEs,

$$
\begin{aligned}
& O_{0}(w) O_{1}(z) \sim-\frac{2}{|w-z|^{2}} O_{1}(z), \\
& O_{0}(w) O_{2}(z) \sim \frac{2}{|w-z|^{2}} O_{2}(z), \\
& O_{1}(w) O_{1}(z) \sim \frac{1}{2} \frac{1}{|w-z|^{4}}-\frac{1}{|w-z|^{2}} O_{0}(z), \\
& O_{2}(w) O_{2}(z) \sim \frac{1}{2} \frac{1}{|w-z|^{4}}+\frac{1}{|w-z|^{2}} O_{0}(z),
\end{aligned}
$$

where terms with scaling dimension bigger than 2 have been ignored. Thus, we have the RG equations,

$$
\begin{aligned}
& \frac{d g_{0}}{d \ell}=\pi\left(g_{1}^{2}-g_{2}^{2}\right), \\
& \frac{d g_{1}}{d \ell}=4 \pi g_{0} g_{1}, \\
& \frac{d g_{2}}{d \ell}=-4 \pi g_{0} g_{2} .
\end{aligned}
$$

Introducing

$$
\frac{1}{2 \pi K}=\frac{1}{2 \pi}+g_{0},
$$

we rewrite the $\mathrm{RG}$ equations as

$$
\begin{aligned}
& \frac{d K}{d \ell}=-2 \pi^{2}\left(K^{2} g_{1}^{2}-g_{2}^{2}\right), \\
& \frac{d g_{1}}{d \ell}=(2-2 K) g_{1}, \\
& \frac{d g_{2}}{d \ell}=\left(2-2 K^{-1}\right) g_{2} .
\end{aligned}
$$

These RG equations are invariant under $\left(K, g_{1}, g_{2}\right) \rightarrow$ $\left(\frac{1}{K}, g_{2}, g_{1}\right)$, and therefore is in accord with the duality transformation of the theory $(K, \varphi, \vartheta) \rightarrow\left(\frac{1}{K}, \vartheta, \varphi\right)$.

\section{APPENDIX E: ORDER PARAMETERS}

In this subsection we investigate possible order parameters of the model. Nonvanishing order parameters detect symmetry-breaking patterns in the model.

(1) The order parameter for the bond spin density wave (bSDW):

$$
\begin{aligned}
& M_{x}=(-1)^{j}\left(c_{j}^{\dagger} \sigma_{x} c_{j+1}+\text { H.c. }\right) \\
& \sim i(-1)^{j}\left(\psi_{L}^{\dagger} e^{-i k_{F} x}+\psi_{R}^{\dagger} e^{i k_{F} x}\right) \sigma_{x} \\
& \times\left(\psi_{L} e^{i k_{F} x}-\psi_{R} e^{-i k_{F} x}\right)+\text { H.c. } \\
& \sim-2 i \psi_{L}^{\dagger} \sigma_{x} \psi_{R}+\text { H.c. } \\
& =\frac{-2 i \eta_{L \downarrow} \eta_{R \uparrow}}{2 \pi \alpha}\left(e^{i \phi_{\downarrow}} e^{i \bar{\phi}_{\uparrow}}-\eta e^{i \phi_{\uparrow}} e^{i \bar{\phi}_{\downarrow}}\right)+\text { H.c. } \\
& =\frac{-2 i \eta_{L \downarrow} \eta_{R \uparrow}}{2 \pi \alpha}\left[e^{i \sqrt{2}\left(\varphi_{c}-\vartheta_{s}\right)}-\eta e^{i \sqrt{2}\left(\varphi_{c}+\vartheta_{s}\right)}\right]+\text { H.c. } \\
& =\frac{-2 i \eta_{L \downarrow} \eta_{R \uparrow}}{\pi \alpha}\left[\cos \sqrt{2}\left(\varphi_{c}-\vartheta_{s}\right)-\eta \cos \sqrt{2}\left(\varphi_{c}+\vartheta_{s}\right)\right] \\
& =\frac{-4 i \eta_{L \downarrow} \eta_{R \uparrow}}{\pi \alpha} \begin{cases}\sin \sqrt{2} \varphi_{c} \sin \sqrt{2} \vartheta_{s} & \eta=1 \\
\cos \sqrt{2} \varphi_{c} \cos \sqrt{2} \vartheta_{s} & \eta=-1 .\end{cases}
\end{aligned}
$$

Similarly,

$$
\begin{aligned}
M_{y} & \sim-2 i \psi_{L}^{\dagger} \sigma_{y} \psi_{R}+\text { H.c. } \\
& =-2\left(\psi_{L \uparrow}^{\dagger} \psi_{R \downarrow}-\psi_{L \downarrow}^{\dagger} \psi_{R \uparrow}\right)+\text { H.c. } \\
& =-\frac{4 i \eta_{L \uparrow} \eta_{R \downarrow}}{\pi \alpha} \begin{cases}\sin \sqrt{2} \varphi_{c} \cos \sqrt{2} \vartheta_{s} & \eta=1 \\
\cos \sqrt{2} \varphi_{c} \sin \sqrt{2} \vartheta_{s} & \eta=-1 .\end{cases}
\end{aligned}
$$

The two expressions are consistent with the fact that the rotation sending $\sigma_{x}$ to $\sigma_{y}$ leads to $\vartheta_{s} \rightarrow \vartheta_{s}+\frac{\pi}{2 \sqrt{2}}$.

(2) The order parameter for the site-local spin density wave (SDW):

$$
\begin{aligned}
& m_{x}=(-1)^{j} c_{j}^{\dagger} \sigma_{x} c_{j} \\
& \sim \psi_{L}^{\dagger} \sigma_{x} \psi_{R}+\text { H.c. }=\psi_{L \uparrow}^{\dagger} \psi_{R \downarrow}+\psi_{L \downarrow}^{\dagger} \psi_{R \uparrow}+\text { H.c. } \\
& =\frac{\eta_{L \uparrow} \eta_{R \downarrow}}{2 \pi \alpha}\left[e^{i \sqrt{2}\left(\varphi_{c}+\vartheta_{s}\right)}-\eta e^{i \sqrt{2}\left(\varphi_{c}-\vartheta_{s}\right)}\right]+\text { H.c. } \\
& =\frac{2 i \eta_{L \uparrow} \eta_{R \downarrow}}{\pi \alpha} \begin{cases}\cos \sqrt{2} \varphi_{c} \sin \sqrt{2} \vartheta_{s} & \eta=1 \\
\sin \sqrt{2} \varphi_{c} \cos \sqrt{2} \vartheta_{s} & \eta=-1,\end{cases} \\
& m_{y}=(-1)^{j} c_{j}^{\dagger} \sigma_{y} c_{j} \\
& \sim \psi_{L}^{\dagger} \sigma_{y} \psi_{R}+\text { H.c. } \\
& =\frac{i \eta_{L \downarrow} \eta_{R \uparrow}}{\pi \alpha}\left[\cos \sqrt{2}\left(\varphi_{c}-\vartheta_{s}\right)+\eta \cos \sqrt{2}\left(\varphi_{c}+\vartheta_{s}\right)\right] \\
& =\frac{2 i \eta_{L \downarrow} \eta_{R \uparrow}}{\pi \alpha} \begin{cases}\cos \sqrt{2} \varphi_{c} \cos \sqrt{2} \vartheta_{s} & \eta=1 \\
\sin \sqrt{2} \varphi_{c} \sin \sqrt{2} \vartheta_{s} & \eta=-1 .\end{cases}
\end{aligned}
$$

The global rotation $R_{z}\left(\frac{\pi}{2}\right)$ from $\sigma_{x}$ to $\sigma_{y}$ exchanges $W_{2}$ and $-W_{2}$, and also sends $M_{x}\left(m_{x}\right)$ to $M_{y}\left(m_{y}\right)$, which means if $M_{x}$ $\left(m_{x}\right)$ condensates for $W_{2}>0$ then $M_{y}\left(m_{y}\right)$ condensate for $W_{2}<0$, and vise versa. 
These are the most possible order parameters, since density orders involving products of oscillations from conjugate fields, and pairing orders breaking the continuous $U(1)$ symmetry are impossible in $(1+1)$ dimensions.

\section{APPENDIX F: MEAN-FIELD THEORY}

\section{Self-consistence of the mean-field theory}

For completeness, we give a self-consistence checking of the mean-field solutions. The self-consistent equation is

$$
\frac{1}{g_{x}}=\frac{2}{L} \sum_{k>0} \frac{\sin ^{2} k}{E_{k}} \tanh \frac{E_{k}}{2 T},
$$

where $E_{k}=\sqrt{2\left(t^{2}+M^{2}\right)+2\left(t^{2}-M^{2}\right) \cos k}$, and we have rescaled $2 k$ to $k$. At zero temperature $T=0$, the equation can be expressed as

$$
\frac{\sqrt{2}}{g_{x}}=\frac{f(\lambda)}{\sqrt{t^{2}+M^{2}}}
$$

where

$$
f(\lambda)=\int_{-\pi}^{\pi} d k \frac{\sin ^{2} k}{\sqrt{1+\lambda \cos k}}, \quad \lambda=\frac{t^{2}-M^{2}}{t^{2}+M^{2}} .
$$

It is observed that $|\lambda| \leqslant 1$, and $f(\lambda)$ slowly depends on $\lambda \in[-1,1]$, roughly $\pi \leqslant f(\lambda) \leqslant 3$.77. Then we have

$$
\frac{1}{g_{x}} \simeq \frac{2}{\sqrt{t^{2}+M^{2}}}
$$

which has nonvanishing real solution

$$
M \simeq 2 g_{x} \sqrt{1-\frac{t}{2 g_{x}}}, \quad \text { if } \quad \frac{t}{2 g_{x}} \lesssim 1 .
$$

\section{The topological invariant}

Since the original lattice has been reduced to be with the lattice constant $2 a$, we need to choose a unit cell consisting of an odd site and its nearest-neighbor even site to diagonalize the Hamiltonian in the real space, which is given by $H=\int d k \psi^{\dagger}(k) \mathcal{H}(k) \psi(k)$, where $\psi^{\dagger}(k)=$ $\left[c_{A \uparrow}^{\dagger}(k), c_{A \downarrow}^{\dagger}(k), c_{B \uparrow}^{\dagger}(k), c_{B \downarrow}^{\dagger}(k)\right]$, with $A$ and $B$ labeling odd and even sublattice, respectively, and

$$
\mathcal{H}(k)=\left(\begin{array}{cc}
0 & \Delta(k) \\
\Delta^{\dagger}(k) & 0
\end{array}\right)
$$

with $\Delta(k)=-t+M \sigma_{x}-\left(t+M \sigma_{x}\right) e^{i 2 a k}$.

The order parameter $M_{x}$ preserves the particle-hole symmetry, which for Eq. (F6) is represented by $\hat{C}=\tau_{3} \hat{\mathcal{K}} \hat{I}$, where $\hat{I}$ denotes the inversion of momenta and $\tau_{3}$ encodes the sign difference for odd and even sublattice under the particle-hole symmetry. The system belongs to the topological class D, and corresponds to the $\mathbb{Z}_{2}$ topological classification. The $\mathbb{Z}_{2}$ topological number is just the Berry phase of the valence states accumulated from encircling the momentum space, $N \equiv \frac{1}{4 \pi i} \oint d k \operatorname{tr}\left[\sigma_{3} \mathcal{H}^{-1}(k) \partial_{k} \mathcal{H}(k)\right] \bmod 2$. For Eq. (F6),

$$
N \equiv \frac{1}{2 \pi i} \oint_{C_{1}} d z \frac{1}{z-r(M)}+\frac{1}{z-1 / r(M)} \bmod 2,
$$

where the loop integration is over the unit circle $C_{1}$, and $r(M)=(t-M) /(t+M)$. Since $r(M)$ is not on $C_{1}$ if $M \neq 0$, either $r(M)$ or $1 / r(M)$ is inside $C_{1}$, but not both, which implies that $N$ is nontrivial for both sides of the dimerization $M>0$ and $M<0$. Thus, the two ends have the half fractional charges, which means that only $\eta=1$ is in accordance with the topological feature of the phase, since

$$
\rho_{L / R}=-\int_{L / R} d x \frac{\sqrt{2}}{\pi} \partial_{x} \varphi_{c}=\mp \frac{1}{2},
$$

only for $\eta=1$ referring to Table I in the main text. Compared with the SSH model, the novelty lies in that the system is in the same $\mathbb{Z}_{2}$ topological phase on both sides of the symmetry breaking $\mathbf{Z}_{2}$.

Since the system is symmetric under $R_{z}\left(\frac{\pi}{2}\right)$ and $W_{2} \rightarrow-W_{2}$, to work out the case for $W_{2}<0$ we merely need to make a global rotation $R_{z}\left(\frac{\pi}{2}\right)$, which leads to $\hat{C} \rightarrow$ $e^{-i \frac{\sigma_{3}}{2} \frac{\pi}{2}} \hat{C} e^{i \frac{\sigma_{3}}{2} \frac{\pi}{2}} \sim \sigma_{3} \tau_{3} \hat{\mathcal{K}} \hat{I}, \Delta(k) \rightarrow e^{-i \frac{\sigma_{3}}{2} \frac{\pi}{2}} \Delta(k) e^{i \frac{\sigma_{3}}{2} \frac{\pi}{2}}=-t+$ $M \sigma_{y}-\left(t+M \sigma_{y}\right) e^{i 2 a k}, \quad M(j) \rightarrow R_{z}(\pi / 2) M(j) R_{z}^{-1}(\pi / 2)=$ $-g_{y}(-1)^{j}\left\langle O_{y}^{+}(j)\right\rangle$, while all topological features are preserved.

\section{APPENDIX G: COLD-ATOM REALIZATION}

In this section, we present technical details for the coldatom realization of the model.

\section{Model Hamiltonian}

For the lattice system, we consider the following Hamiltonian composed of three terms,

$$
H=H_{0}+H_{i 1}+H_{i 2} \text {. }
$$

The first term describes the coupling between the states $|g\rangle$ and $|e\rangle$

$$
H_{0}=\Omega \int d x\left[e_{1}^{\dagger}(x) g_{2}(x)+e_{2}^{\dagger}(x) g_{1}(x)\right]+\text { H.c. },
$$

where $\Omega$ is the coupling strength, and $\lambda_{v}$ with $v=1,2$ are the annihilation operator of the corresponding $\left|\lambda_{\nu}\right\rangle$ state. The second and third terms describes two types of interactions given by

$$
\begin{aligned}
& H_{i 1}=U_{1} \int d x e_{1}^{\dagger}(x) g_{1}^{\dagger}(x) e_{2}(x) g_{2}(x)+\text { H.c. }, \\
& H_{i 2}=U_{2} \int d x g_{2}^{\dagger}(x) e_{1}^{\dagger}(x) g_{1}(x) e_{2}(x)+\text { H.c. },
\end{aligned}
$$

where $U_{1 / 2}$ denote the interaction strength.

To investigate the physics of the lattice system, we use the tight-binding approximation by expanding $\lambda_{v}$ by the Wannier wave functions $W(x)$ :

$$
\begin{aligned}
& \lambda_{1}(x)=\sum_{j} W\left(x-x_{j}\right) \lambda_{1, j}, \\
& \lambda_{2}(x)=\sum_{j^{\prime}} W\left(x-x_{j^{\prime}}\right) \lambda_{2, j^{\prime}},
\end{aligned}
$$

where $x_{j^{\prime}}=x_{j}+a / 2$ due to the spin-dependent lattice configuration. We rearrange the site index $j$ and $j^{\prime}$ by representing $j$ as the odd site index while $j^{\prime}$ as the even site one. Then the 
three terms in Hamiltonian Eq. (G1) are rewritten as

$$
\begin{aligned}
& H_{0}=-t \sum_{j}\left[e_{1, j}^{\dagger}\left(g_{2, j}+g_{2, j-1}\right)+e_{2, j}^{\dagger}\left(g_{1, j}+g_{1, j+1}\right)\right]+\text { H.c., } \\
& H_{i 1}=W_{1} \sum_{j} e_{1, j}^{\dagger} g_{1, j}^{\dagger}\left(e_{2, j} g_{2, j}+e_{2, j-1} g_{2, j-1}\right)+\text { H.c., } \\
& H_{i 2}=W_{2} \sum_{j}\left(g_{2, j}^{\dagger} e_{1, j}^{\dagger} g_{1, j} e_{2, j}+g_{2, j}^{\dagger} e_{1, j+1}^{\dagger} g_{1, j+1} e_{2, j}\right)+\text { H.c., }
\end{aligned}
$$

with

$$
\begin{aligned}
t & =-\Omega \int d x W^{*}(x) W(x+a / 2), \\
W_{1,2} & =U_{1,2} \int d x|W(x) W(x+a / 2)|^{2} .
\end{aligned}
$$

We assign the following operator correspondences:

$g_{1} \rightarrow c_{j \downarrow}, \quad g_{2} \rightarrow c_{j+1 \uparrow}, \quad e_{1} \rightarrow c_{j \uparrow}, \quad e_{2} \rightarrow c_{j+1 \downarrow}$,

from which one can check that the Hamiltonian of Eq. (2) in the main text is exactly simulated.

\section{The optical Feshbach resonance}

As introduced in the main text, we use the optical Feshbach resonance to realize the $W_{1}$ interaction term. The optical Feshbach resonance can be described by a two-channel model. We denote the two open channels $\left|\operatorname{op}_{1}\right\rangle=\left|g_{1}, e_{1}\right\rangle$ and $\left|\mathrm{op}_{2}\right\rangle=\left|g_{2}, e_{2}\right\rangle$, and the the closed channel $|\mathrm{cl}\rangle=\left|e_{1}, e_{2}\right\rangle$. As illustrated in Fig. 4(a), the Hamiltonian in the base of $\Psi=\left(|\mathrm{cl}\rangle,\left|\mathrm{op}_{1}\right\rangle,\left|\mathrm{op}_{2}\right\rangle\right)^{T}$ is expressed as

$$
\left(\begin{array}{ccc}
H_{\mathrm{cl}} & \Omega_{1} & \Omega_{2} \\
\Omega_{1}^{*} & H_{\mathrm{op}_{1}} & 0 \\
\Omega_{2}^{*} & 0 & H_{\mathrm{Op}_{2}}
\end{array}\right) \Psi=E \Psi,
$$

where the Hamiltonians for each channel are given by

$$
H_{\mathrm{cl}}=\frac{k^{2}}{m}, \quad H_{\mathrm{op}_{i=1,2}}=\frac{k^{2}}{m}-\delta_{i} .
$$

Here, $m$ is the atomic mass, $k$ is the momentum, and $\delta_{i}$ is the detuning of each open channel compared with the closed channel. For the closed channel, we have

$$
\left(E-H_{\mathrm{cl}}\right)|\mathrm{cl}\rangle=\sum_{i} \Omega_{i}^{*}\left|\mathrm{op}_{i}\right\rangle .
$$

For the bound state of the closed channel, it gives

$$
H_{\mathrm{cl}}|\mathrm{cl}\rangle=E_{B}|B\rangle \Rightarrow|B\rangle=\sum_{j} \frac{\Omega_{j}^{*}}{E-E_{B}}\left|\mathrm{op}_{j}\right\rangle .
$$

For the open channels, we have

$$
\left(E-H_{\mathrm{op}_{i}}\right)\left|\mathrm{op}_{i}\right\rangle=\Omega_{i}|B\rangle .
$$

Inserting Eq. (G10) into Eq. (G11), we obtain

$$
\begin{aligned}
& \left|\mathrm{op}_{1}\right\rangle=\frac{\Omega_{1} \Omega_{2}^{*}}{\left(E-E_{B}\right)\left(E-H_{\mathrm{op}_{1}}\right)-\left|\Omega_{1}\right|^{2}}\left|\mathrm{op}_{2}\right\rangle, \\
& \left|\mathrm{op}_{2}\right\rangle=\frac{\Omega_{2} \Omega_{1}^{*}}{\left(E-E_{B}\right)\left(E-H_{\mathrm{op}_{2}}\right)-\left|\Omega_{2}\right|^{2}}\left|\mathrm{op}_{1}\right\rangle .
\end{aligned}
$$
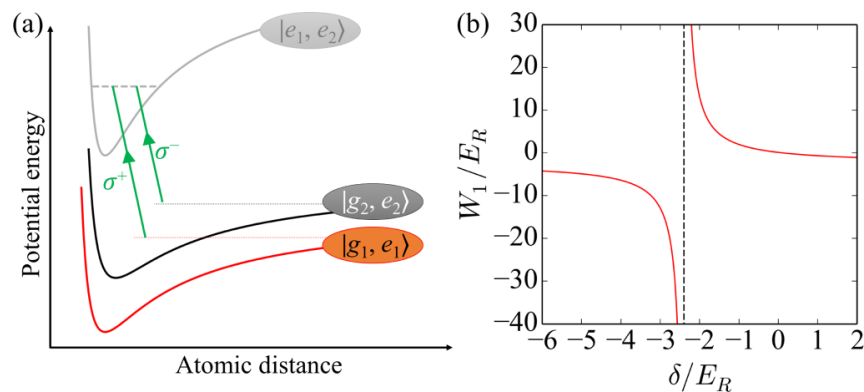

FIG. 4. (a) Illustration of the optical Feshbach resonance. The two open channels $\left|g_{i}, e_{i}\right\rangle(i=1,2)$ are coupled to the closed channel $\left|e_{1}, e_{2}\right\rangle$ via circularly polarized lasers. (b) The interaction strength $W_{1}$ as a function of the detuning $\delta$. The black dashed line marks the resonant point $\delta_{\text {res }}=E_{B}-W^{2} / E_{B}$. We set $W=0.5 E_{R}$ and $E_{B}=0.1 E_{R}$.

The Lippmann-Schwinger equation for each open channel reads

$$
\left|\mathrm{op}_{i}\right\rangle=|k\rangle+G_{i} V_{\mathrm{eff}}^{(i)}\left|\mathrm{op}_{i}\right\rangle,
$$

where $|k\rangle$ is the wave function of free atoms in the scattering process, $G_{i}^{-1}=E-H_{\mathrm{op}_{i}}+i 0^{+}$is the Green's function for each open channel, and

$V_{\mathrm{eff}}^{(1)}=\frac{\Omega_{1} \Omega_{2}^{*}}{\left(E-E_{B}\right)-\frac{\left|\Omega_{1}\right|^{2}}{E-E_{B}+\delta_{1}}}, \quad V_{\mathrm{eff}}^{(2)}=\frac{\Omega_{2} \Omega_{1}^{*}}{\left(E-E_{B}\right)-\frac{\left|\Omega_{2}\right|^{2}}{E-E_{B}+\delta_{2}}}$.

For simplicity, if the Raman transition is resonant, i.e., $\delta_{1}=$ $\delta_{2}=\delta$, and we tune the laser field strength $\left|\Omega_{1}\right| \approx\left|\Omega_{2}\right| \approx W$, then the interacting Hamiltonian is written as

$$
H_{\mathrm{i} 1}=W_{1}\left|\mathrm{op}_{1}\right\rangle\left\langle\mathrm{op}_{2}\right|+\text { H.c., }
$$

with the controllable strength

$$
W_{1}=\frac{W^{2}}{\left(E-E_{B}\right)-\frac{W^{2}}{E-E_{B}+\delta}} .
$$

It is noted that: (i) We can tune the Raman detuning $\delta$ and Raman laser field strength $W$ (rather than the bound state energy $E_{B}$ that is usually used in magnetic Feshbach resonance), to change the strength $W_{1}$ of the $H_{i 1}$ term. (ii) In contrast, the strength $W_{2}$ of the $H_{i 2}$ term can be still tuned by the magnetic shift to its bound state energy. Based on these facts, the interaction strengths, $W_{1}$ and $W_{2}$, can be independently tuned.

In real experiments, for Eq. (G16), $E \sim 0$ and $E_{B} \sim(B-$ $B_{0}$ ), where $B$ is the magnetic field strength acting on the closed channel bound state, and $B_{0}$ is the strength at the resonant point of the Feshbach resonance. For ${ }^{173} \mathrm{Yb}$, as an example, the optical lattice laser wavelength is $\lambda \approx 759 \mathrm{~nm}$ [33], thus the lattice recoil energy, $E_{R}=h^{2} /\left(2 m \lambda_{L}^{2}\right) \approx$ $2.043 \mathrm{kHz} \times 2 \pi \hbar$, which can be chosen as unit. If we set $E_{B}=0.1 E_{R}$ and $W=0.5 E_{R}$, then the interaction strength $W_{1}$ as a function of $\delta$ is shown in Fig. 4(b). Usually, the tunneling $t \sim 0.1 E_{R}$ for the 1D tight-binding model. From Fig. 4(b), we can see that the parameter range $t<\left|W_{1}\right|<10 t$ is achievable. 
[1] M. Z. Hasan and C. L. Kane, Rev. Mod. Phys. 82, 3045 (2010).

[2] X.-L. Qi and S.-C. Zhang, Rev. Mod. Phys. 83, 1057 (2011).

[3] T. Senthil, Annu. Rev. Condens. Matter Phys. 6, 299 (2015).

[4] C.-K. Chiu, J. C. Y. Teo, A. P. Schnyder, and S. Ryu, Rev. Mod. Phys. 88, 035005 (2016).

[5] X. Chen, Z.-C. Gu, and X.-G. Wen, Phys. Rev. B 83, 035107 (2011).

[6] F. Pollmann, E. Berg, A. M. Turner, and M. Oshikawa, Phys. Rev. B 85, 075125 (2012).

[7] L. Fidkowski and A. Kitaev, Phys. Rev. B 83, 075103 (2011).

[8] X. Chen, Z.-C. Gu, Z.-X. Liu, and X.-G. Wen, Science 338, 1604 (2012).

[9] Y. X. Zhao and Z. D. Wang, Phys. Rev. Lett. 110, 240404 (2013).

[10] Y. X. Zhao, A. P. Schnyder, and Z. D. Wang, Phys. Rev. Lett. 116, 156402 (2016).

[11] D. Hsieh, D. Qian, L. Wray, Y. Xia, Y. S. Hor, R. Cava, and M. Z. Hasan, Nature 452, 970 (2008).

[12] M. König, S. Wiedmann, C. Brüne, A. Roth, H. Buhmann, L. W. Molenkamp, X.-L. Qi, and S.-C. Zhang, Science 318, 766 (2007).

[13] A. P. Schnyder, S. Ryu, A. Furusaki, and A. W. W. Ludwig, Phys. Rev. B 78, 195125 (2008).

[14] A. Kitaev, in Advances in Theoretical Physics: Landau Memorial Conference, edited by V. Lebedev and M. Feigelman, AIP Conf. Proc. No. 1134 (AIP, New York, 2009), p. 22.

[15] L. Landau and E. Lifschitz, Phys. Z. Sowjetunion 11, 545 (1937).

[16] R. E. Peierls, Quantum Theory of Solids (Oxford University Press, Oxford, 1955), Vol. 23.
[17] W. P. Su, J. R. Schrieffer, and A. J. Heeger, Phys. Rev. Lett. 42, 1698 (1979).

[18] A. J. Heeger, S. Kivelson, J. R. Schrieffer, and W. P. Su, Rev. Mod. Phys. 60, 781 (1988).

[19] J. Goldstone and F. Wilczek, Phys. Rev. Lett. 47, 986 (1981).

[20] R. Jackiw and C. Rebbi, Phys. Rev. D 13, 3398 (1976).

[21] G. Japaridze and S. Sarkar, Eur. Phys. J. B 28, 139 (2002).

[22] M. Cheng and H.-H. Tu, Phys. Rev. B 84, 094503 (2011).

[23] T. Giamarchi, Quantum Physics in One Dimension (Oxford University Press, Oxford, 2004).

[24] J. Voit, Phys. Rev. B 45, 4027 (1992).

[25] M. Tsuchiizu and A. Furusaki, Phys. Rev. Lett. 88, 056402 (2002).

[26] S. Ejima and S. Nishimoto, Phys. Rev. Lett. 99, 216403 (2007).

[27] O. Mandel, M. Greiner, A. Widera, T. Rom, T. W. Hänsch, and I. Bloch, Phys. Rev. Lett. 91, 010407 (2003).

[28] M. Aidelsburger, M. Atala, M. Lohse, J. T. Barreiro, B. Paredes, and I. Bloch, Phys. Rev. Lett. 111, 185301 (2013).

[29] H. Miyake, G. A. Siviloglou, C. J. Kennedy, W. C. Burton, and W. Ketterle, Phys. Rev. Lett. 111, 185302 (2013).

[30] A. V. Gorshkov, M. Hermele, V. Gurarie, C. Xu, P. S. Julienne, J. Ye, P. Zoller, E. Demler, M. D. Lukin, and A. Rey, Nat. Phys. 6, 289 (2010)

[31] G. Pagano, M. Mancini, G. Cappellini, L. Livi, C. Sias, J. Catani, M. Inguscio, and L. Fallani, Phys. Rev. Lett. 115, 265301 (2015).

[32] K. Enomoto, K. Kasa, M. Kitagawa, and Y. Takahashi, Phys. Rev. Lett. 101, 203201 (2008).

[33] G. Cappellini, M. Mancini, G. Pagano, P. Lombardi, L. Livi, M. Siciliani de Cumis, P. Cancio, M. Pizzocaro, D. Calonico, F. Levi, C. Sias, J. Catani, M. Inguscio, and L. Fallani, Phys. Rev. Lett. 113, 120402 (2014). 